\title{
Theoretical investigation of moiré patterns in quantum images
}

\author{
M. P. Almeida, P. H. Souto Ribeiro \\ Instituto de Física - Universidade Federal do Rio de Janeiro \\ Caixa Postal 68528, Rio de Janeiro - RJ, 21941-972, \\ Brazil. and J. A. O. Huguenin, A. Z. Khoury \\ Instituto de Física - Universidade Federal Fluminense \\ Niterói - RJ, 24210-340, Brazil.
}

(Dated: November 17, 2018)

\begin{abstract}
Moiré patterns are produced when two periodic structures with different spatial frequencies are superposed. The transmission of the resulting structure gives rise to spatial beatings which are called moiré fringes. In classical optics, the interest in moiré fringes comes from the fact that the spatial beating given by the frequency difference gives information about details(high spatial frequency) of a given spatial structure. We show that moiré fringes can also arise in the spatial distribution of the coincidence count rate of twin photons from the parametric down-conversion, when spatial structures with different frequencies are placed in the path of each one of the twin beams. In other words, we demonstrate how moiré fringes can arise from quantum images.
\end{abstract}

PACS numbers: 42.50.Ar; 42.50.St; 42.50.Lc 
Quantum image is the name used by several authors of scientific reports, for the spatial correlations of a light source possessing some non-classical property. Even though in some cases the calculated or measured pattern has a classical counterpart, it is known that some quantum images are actually spatial patterns with no classical counterpart. The activity in this field has increased over the last ten years [1].

Besides the academic interest in demonstrating differences between classical and quantum descriptions of light, it would be of great importance to demonstrate the possibility of some practical application. One way towards practical applications is the combination between quantum images and other known physical processes. One example is the use of quantum spatial correlations for improving the resolution of imaging systems. Apodization in correlated images 2] and the so called quantum lithography, whose fundamental principle was

demonstrated by Fonseca et al. [3] and has received this name afterwards [4, 5, 6]. Though promising, none of these practical applications has overcome all the technical difficulties in actually replacing classical systems with some advantage. In the case of the quantum lithography, for instance, no substrate has been found to efficiently absorb two photons.

In this report, we would like to introduce the theoretical analysis for other promising practical application for quantum images. It has been demonstrated experimentally [7] that it is possible to observe moiré patterns in quantum images, for two fundamentally different experimental configurations. One configuration is based on the transfer of the angular spectrum from the pump to the twin photons 8] and other is based directly on the nonlocal correlations between signal and idler photons[10]. In both cases, the calculations demonstrate that the coincidence count rate depends on a product of functions describing the periodic structures used. A good agreement between the theory presented here and the experimental results of Ref. 7]] is achieved

\section{PUMP-IDLER SETUP}

In this section we will calculate the coincidence profiles for the two configurations mentioned above. We start from the state of the field generated by spontaneous parametric down-conversion for a thin crystals, in the monochromatic and paraxial approximation [8]

$$
|\psi\rangle=c_{1}|v a c\rangle+c_{2} \int d_{s} \int d q_{i} \mathcal{V}_{z_{0}}\left(q_{s}+q_{i}\right)\left|1, q_{s}\right\rangle\left|1, q_{i}\right\rangle
$$




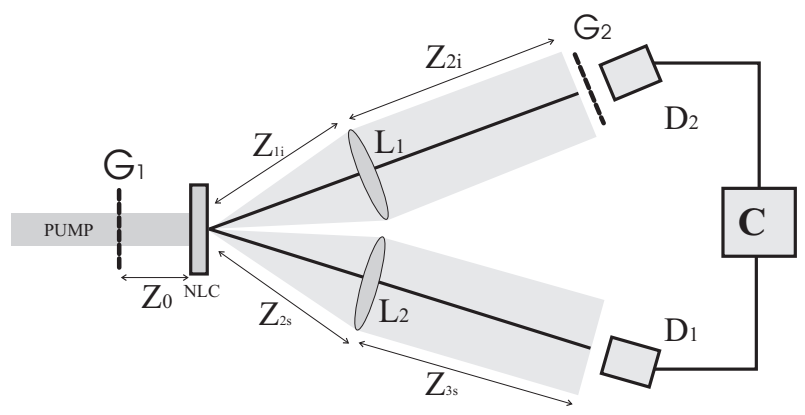

FIG. 1: Pump-Idler setup.

where $c_{1}$ and $c_{2}$ are constant coefficients, $\left|1, q_{s}\right\rangle,\left|1, q_{i}\right\rangle$ are the single photon Fock states with transverse wave vectors corresponding to the down-converted signal and idler modes, respectively. $|v a c\rangle$ represents the vacuum state of the electromagnetic field. $\mathcal{V}_{z_{0}}\left(q_{s}+q_{i}\right)$, with $q_{p}=q_{s}+q_{i}$, is the angular spectrum of the pump, at the crystal position. In terms of the angular spectrum immediately after the object $\mathcal{V}_{0}\left(q_{p}\right)$, we can write:

$$
\mathcal{V}_{z_{0}} \propto \mathcal{V}_{0} \exp \left[-i \frac{q_{p}^{2} z_{1}}{2 k_{p}}\right]
$$

The coincidence count rate $C\left(\rho_{s}, \rho_{i}\right)$, in the transverse plane of the detectors, is proportional to the fourth-order correlation function $G^{(2,2)}\left(\rho_{s}, \rho_{i}\right)$, given by:

$$
\begin{array}{r}
G^{(2,2)}\left(\rho_{s}, \rho_{i}\right)=\left\langle\psi\left|\hat{E}^{(-)}\left(\rho_{s}\right) \hat{E}^{(-)}\left(\rho_{i}\right) \hat{E}^{(+)}\left(\rho_{s}\right) \hat{E}^{(+)}\left(\rho_{i}\right)\right| \psi\right\rangle= \\
\left|\left\langle v a c\left|\hat{E}^{(+)}\left(\rho_{s}\right) \hat{E}^{(+)}\left(\rho_{i}\right)\right| \psi\right\rangle\right|^{2}
\end{array}
$$

First we will consider the setup showed in the Fig. 1. The first grating is placed in the pump beam and its image is transferred to the transverse coincidence distribution between the signal and idler beams, with the aid of lenses L1 and L2 [9]. The second grating is placed in the idler beam. The resulting moiré pattern is produced by the superposition of the conditional image and the second grating. We call this configuration pump-idler setup. Assuming that $\mathcal{A}_{2}$ is the transmission function of the grating $G_{2}$, we can write the field operator for the idler mode as:

$$
\begin{array}{r}
\hat{E}_{i}^{(+)}\left(\rho_{i}\right)=\mathcal{A}_{2}\left(\rho_{i}\right) \int d \rho_{i}^{\prime \prime} \int d \rho_{i}^{\prime} \int d q_{i}^{\prime} \hat{a}\left(q_{i}^{\prime}\right) \exp \left[i q_{i} \cdot \rho^{\prime}{ }_{i}\right] \\
\times \exp \left[i\left(\left|\rho^{\prime \prime}{ }_{i}-\rho^{\prime}{ }_{i}\right|^{2} \frac{k_{i}}{2 z_{1} i}\right)\right] \mathcal{T}\left(\rho^{\prime \prime}{ }_{i}\right) \exp \left[i\left(\left|\rho_{i}-\rho^{\prime \prime}{ }_{i}\right|^{2} \frac{k_{i}}{2 z_{2} i}\right)\right],
\end{array}
$$


where $k_{i}$ is the wave number of the idler beam. $\mathcal{T}_{1}\left(\rho_{i}^{\prime \prime}\right)$ is the transmission function of the lens $L_{1}$, given by:

$$
\mathcal{T}_{1}\left(\rho_{i}^{\prime \prime}\right)=\exp \left(-i \frac{\rho_{i}^{\prime 2} k_{i}}{2 f}\right)
$$

The field operator for the signal mode, at the detection plane can be written as:

$$
\begin{aligned}
\hat{E}_{s}^{(+)}\left(\rho_{s}\right)= & \int d \rho_{s}^{\prime \prime} \int d \rho_{s}^{\prime} \int d q_{s}^{\prime} \hat{a}\left(q_{s}^{\prime}\right) \exp \left[i q_{s}^{\prime} \cdot \rho^{\prime}{ }_{s}\right] \\
& \times \exp \left[i\left(\left|\rho^{\prime \prime}{ }_{s}-\rho^{\prime}{ }_{s}\right|^{2} \frac{k_{s}}{2 z_{1} s}\right)\right] \mathcal{T}\left(\rho^{\prime \prime}{ }_{s}\right) \\
& \times \exp \left[i\left(\left|\rho_{s}-\rho^{\prime \prime}{ }_{s}\right|^{2} \frac{k_{s}}{2 z_{2} s}\right)\right] .
\end{aligned}
$$

Using Eqs.(11)-(6) we can calculate the fourth order correlation function:

$$
\begin{aligned}
& \left\langle v a c\left|\hat{E}^{(+)}\left(\rho_{s}\right) \hat{E}^{(+)}\left(\rho_{i}\right)\right| \psi\right\rangle= \\
& \mathcal{A}_{2}\left(\rho_{i}\right) \int d \rho^{\prime \prime}{ }_{s} \int d \rho^{\prime}{ }_{s} \int d \rho^{\prime \prime}{ }_{i} \int d \rho^{\prime}{ }_{i} \int d q^{\prime} \int d q_{i}{ }_{i} \mathcal{V}_{z_{0}}\left(q^{\prime}{ }_{s}+q_{i}^{\prime}\right) \\
& \times \exp \left[i\left(\left|\rho^{\prime \prime}{ }_{s}-\rho^{\prime}{ }_{s}\right|^{2} \frac{k_{s}}{2 z_{1 s}}\right)\right] \exp \left(-i \frac{\rho_{s}^{\prime \prime 2} k_{s}}{2 f}\right) \\
& \times \exp \left[i\left(\left|\rho_{s}-\rho^{\prime \prime}{ }_{s}\right|^{2} \frac{k_{s}}{2 z_{2 s}}\right)\right] \exp \left(i q_{s}^{\prime} \rho_{s}^{\prime}\right) \\
& \times \exp \left[i\left(\left|\rho^{\prime \prime}{ }_{i}-\rho_{i}^{\prime}\right|^{2} \frac{k_{i}}{2 z_{1}}\right)\right] \exp \left(-i \frac{\rho_{i}^{\prime \prime 2} k_{i}}{2 f}\right) \\
& \times \exp \left[i\left(\left|\rho_{s}-\rho^{\prime \prime}{ }_{i}\right|^{2} \frac{k_{i}}{2 z_{2 i}}\right)\right] \exp \left(i q_{i}^{\prime} \rho_{i}^{\prime}\right) \text {. }
\end{aligned}
$$

Performing the integrals over the variables $\rho^{\prime \prime}{ }_{s}, \rho^{\prime \prime}{ }_{i}, \rho_{s}^{\prime}$ and $\rho_{i}^{\prime}$, results in the following expression:

$$
\begin{array}{r}
\left\langle\operatorname{vac}\left|\hat{E}^{(+)}\left(\rho_{s}\right) \hat{E}^{(+)}\left(\rho_{i}\right)\right| \psi\right\rangle= \\
\mathcal{A}_{2}\left(\rho_{i}\right) \int d q^{\prime}{ }_{s} \int d q^{\prime}{ }_{i} v_{z_{0}}\left(q^{\prime}{ }_{s}+q^{\prime}{ }_{i}\right) \\
\times \exp \left[i \rho_{s}^{2}\left(\frac{k_{s}}{2 z_{2} s}+\frac{k_{s}^{2}}{4 \alpha_{s}} \frac{1}{z_{2}^{2}}\right)\right] \exp \left[i \rho_{i}^{2}\left(\frac{k_{s}}{2 z_{2} i}+\frac{k_{i}^{2}}{4 \alpha_{i}} \frac{1}{z_{i}^{2}}\right)\right] \\
\times \exp \left[i q_{s}^{2}\left(\frac{1}{4 \alpha_{s}}-\frac{z 1 s}{2 k_{s}}\right)\right] \exp \left[i q_{i}^{2}\left(\frac{1}{4 \alpha_{i}}-\frac{z 1 i}{2 k_{i}}\right)\right] \\
\times \exp \left(\frac{-i k_{s}}{2 z_{2 s} \alpha_{s}}\right) \exp \left(\frac{-i k_{i}}{2 z_{2 i} \alpha_{i}}\right),
\end{array}
$$

where the $\alpha_{j}, j=i, s$ are given by:

$$
\alpha_{j}=\frac{k_{j}}{2 f}-\frac{k_{j}}{2 z_{2 j}} .
$$


Terms in the Eq.(8) depending only on the variables $\rho_{s}$ and $\rho_{i}$, do not contribute to the integrals over $q_{s}$ and $q_{i}$, and can be omitted, because the coincidence count rate depends on its square modulus. Assuming that the down-converted fields have the same frequency, and the distances from the crystal to the lenses and from the lenses to the detectors are the same for both modes, we have:

$$
\begin{array}{r}
k_{s}=k_{i}=k ; \\
\alpha_{s}=\alpha_{i} .
\end{array}
$$

Using the thin lens law $\frac{1}{f}=\frac{1}{I}+\frac{1}{O}$, with $O=z_{0}+z_{1}$ and $I=z_{2}$, we can rewrite Eq.(17) as:

$$
\begin{array}{r}
\left\langle v a c\left|\hat{E}^{(+)}\left(\rho_{s}\right) \hat{E}^{(+)}\left(\rho_{i}\right)\right| \psi\right\rangle= \\
\mathcal{A}_{2}\left(\rho_{i}\right) \int d q^{\prime}{ }_{s} \int d q^{\prime}{ }_{i} v_{z_{0}}\left(q^{\prime}{ }_{s}+q^{\prime}{ }_{i}\right) \\
\times \exp \left[i \mathcal{B}\left(q_{s}^{2}+q_{i}^{2}\right)\right] \exp \left[i\left(q_{s}^{2}+q_{i}^{2}\right)\right],
\end{array}
$$

where $\mathcal{B}$ is given by:

$$
\mathcal{B}=\frac{1}{4 \alpha}-\frac{z_{1}}{k}
$$

Introducing the relatives variables

$$
\begin{aligned}
& u=q_{s}+q_{i}, \\
& v=q_{s}-q_{i},
\end{aligned}
$$

we can rewrite Eq. (12) as:

$$
\begin{array}{r}
\left\langle v a c\left|\hat{E}^{(+)}\left(\rho_{s}\right) \hat{E}^{(+)}\left(\rho_{i}\right)\right| \psi\right\rangle= \\
\mathcal{A}_{2}\left(\rho_{i}\right) \int d u \int d v \mathcal{V}_{z_{0}}(u) \exp \left[i \mathcal{B}\left(\frac{u^{2}+v^{2}}{2}\right)\right] \\
\times \exp \left[-i \frac{O}{I}\left(\frac{u+v}{2} \cdot \rho_{s}+\frac{u-v}{2} \cdot \rho_{i}\right)\right]= \\
\int d u \mathcal{V}_{0}(u) \exp \left[-i \frac{O}{I} u \cdot\left(\frac{\rho_{i}+\rho_{s}}{2}\right)\right] \\
\times \int d v \exp \left[i \frac{\mathcal{B}}{2} v^{2}\right] \exp \left[-i \frac{O}{I} v \cdot\left(\frac{\rho_{i}-\rho_{s}}{2}\right)\right] .
\end{array}
$$

The integral over $u$ is the Fourier Transform of the angular spectrum of pump beam, while the integral over $v$ will add a phase factor, which will be equal to one, when we take the square 


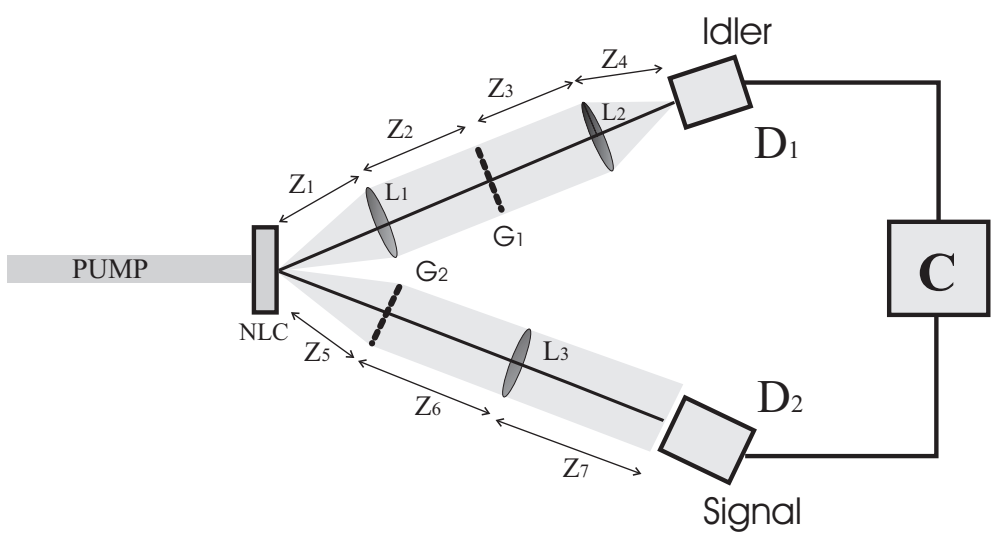

FIG. 2: Idler-Signal setup.

modulus. Solving these integrals we have the following expression for the coincidence-count rate:

$$
C\left(\rho_{s}, \rho_{i}\right) \propto\left|\mathcal{A}_{2}\left(\rho_{i}\right) \mathcal{W}_{p}\left[\frac{-\left(\rho_{s}+\rho_{i}\right)}{2}\right]\right|^{2} .
$$

$\mathcal{W}_{p}=\mathcal{A}_{1} \mathcal{E}_{0}$ is the field distribution of the pump beam immediately after the grating plane, and $\mathcal{E}_{0}$ is its amplitude before the mask. Assuming that the pump beam is a plane wave, $\mathcal{E}_{0}=$ const. Thus

$$
C\left(\rho_{s}, \rho_{i}\right) \propto\left|\mathcal{A}_{2}\left(\rho_{i}\right) \mathcal{A}_{1}\left[\frac{-\left(\rho_{s}+\rho_{i}\right)}{2}\right]\right|^{2} .
$$

We see that $C\left(\rho_{s}, \rho_{i}\right)$ is given by the product of the transmission functions of the gratings.

\section{IDLER-SIGNAL SETUP}

Now, we will calculate the coincidence count rate for the setup showed in Fig 2. In this case, the two gratings are placed in the signal and idler beams. The resulting moiré pattern is produced by the superposition of the correlated images of these objects. We call this configuration idler-signal setup. The field operator for the idler mode, propagated from crystal to the detection plane, is given by

$$
\begin{array}{r}
\hat{E}_{i}^{(+)}\left(\rho_{i}, Z_{i}\right)=\int d \rho_{i}^{\prime} \int d \rho^{\prime \prime}{ }_{i} \int d \rho^{\prime \prime \prime}{ }_{i} \int d \rho^{\prime \prime \prime \prime} \int d q^{\prime \prime \prime \prime}{ }_{i} \hat{a}\left(q^{\prime \prime \prime \prime}{ }_{i}\right) \exp \left[i q^{\prime \prime \prime \prime}{ }_{i} \cdot \rho^{\prime \prime \prime \prime}{ }_{i}\right] \\
\times \exp \left[i\left(\left|\rho_{i}-\rho^{\prime}{ }_{i}\right|^{2} \frac{k_{i}}{2 z_{1 i}}\right)\right] \mathcal{T}_{1}\left(\rho_{i}^{\prime}\right) \exp \left[i\left(\left|\rho_{i}^{\prime}-\rho^{\prime \prime}{ }_{i}\right|^{2} \frac{k_{i}}{2 z_{2 i}}\right)\right] \mathcal{A}_{1}\left(\rho^{\prime \prime}{ }_{i}\right) \\
\quad \times \exp \left[i\left(\left|\rho^{\prime \prime}{ }_{i}-\rho^{\prime \prime \prime}{ }_{i}\right|^{2} \frac{k_{i}}{2 z_{3 i}}\right)\right] \mathcal{T}_{2}\left(\rho^{\prime \prime \prime}{ }_{i}\right) \exp \left[i\left(\left|\rho^{\prime \prime \prime}{ }_{i}-\rho^{\prime \prime \prime \prime \prime}{ }_{i}\right|^{2} \frac{k_{i}}{2 z_{4 i}}\right)\right],
\end{array}
$$


where $\mathcal{T}_{1}\left(\rho^{\prime \prime}{ }_{i}\right)$ and $\mathcal{T}_{2}\left(\rho^{\prime \prime \prime}{ }_{i}\right)$ are the transmission functions of the lenses $L_{1}$ and $L_{2} . \mathcal{A}_{1}\left(\rho^{\prime \prime}{ }_{i}\right)$ is the transmission function of the grating $G 1$. Solving the integral over $\rho^{\prime}, \rho^{\prime \prime \prime}$ and $\rho^{\prime \prime \prime \prime}$, assuming that $z_{1}=z_{4}=f$ and $z_{2}=z_{3}=2 f$, where $f$ is the focal length of the lenses $L_{1}$ and $L_{2}$, it is possible reduce this expression to:

$$
\begin{aligned}
& \hat{E}_{i}^{(+)}\left(\rho_{i}, Z_{i}\right)=\int d q^{\prime \prime \prime \prime}{ }_{i} \hat{a}\left(q^{\prime \prime \prime \prime}{ }_{i}\right) \int d \rho^{\prime \prime}{ }_{i} \mathcal{A}_{1}\left(\rho^{\prime \prime}{ }_{i}\right) \\
& \quad \times \exp \left[i \frac{q_{i}^{\prime \prime \prime \prime} f}{2 k_{i}}\right] \exp \left[-i \rho^{\prime \prime}{ }_{i}\left(\frac{\rho_{i} k_{i}}{f}+q^{\prime \prime \prime \prime \prime}{ }_{i}\right)\right] .
\end{aligned}
$$

Again, we have omitted the term that depends only on the variables $\rho_{i}$.

For the signal mode the field operator is given by:

$$
\begin{array}{r}
\hat{E}_{s}^{(+)}\left(\rho_{s}, Z_{s}\right)=\int d \rho_{i}^{\prime} \int d \rho^{\prime \prime}{ }_{s} \int d \rho^{\prime \prime \prime}{ }_{s} \int d q^{\prime \prime \prime}{ }_{i} \hat{a}\left(q^{\prime \prime \prime \prime}{ }_{i}\right) \exp \left[i q^{\prime \prime \prime}{ }_{i} \cdot \rho^{\prime \prime \prime}{ }_{i}\right] \\
\times \exp \left[i\left(\left|\rho_{s}-\rho^{\prime}{ }_{s}\right|^{2} \frac{k_{s}}{2 z_{5 s}}\right)\right] \mathcal{A}_{2}\left(\rho_{i}^{\prime}\right) \exp \left[i\left(\left|\rho^{\prime}{ }_{s}-\rho_{{ }^{\prime \prime}}{ }_{s}\right|^{2} \frac{k_{s}}{2 z_{6 s}}\right)\right] \mathcal{T}_{3}\left(\rho^{\prime \prime}{ }_{i}\right) \\
\times \exp \left[i\left(\left|\rho^{\prime \prime}{ }_{s}-\rho^{\prime \prime \prime}{ }_{s}\right|^{2} \frac{k_{s}}{2 z_{7 s}}\right)\right],
\end{array}
$$

where $\mathcal{A}_{2}\left(\rho_{i}^{\prime}\right)$ and $\mathcal{T}_{3}\left(\rho^{\prime \prime}\right)$ are transmission functions of the grating $G_{2}$ and of the lens $L_{4}$, respectively. $z_{5}=f$ and $z_{6}=z_{7}=2 f$, where $\mathrm{f}$ is the focal length of the lens $L_{4}$. Performing the integrals over the variables $\rho_{s}^{\prime}, \rho^{\prime \prime}{ }_{s}$ and $\rho^{\prime \prime \prime}{ }_{s}$, we have

$$
\hat{E}_{s}^{(+)}\left(\rho_{s}, Z_{s}\right)=\mathcal{A}_{2}\left(\rho_{s}^{\prime}\right) \times \int d q^{\prime \prime \prime \prime}{ }_{s} \hat{a}\left(q^{\prime \prime \prime}{ }_{s}\right) \exp \left[-i q^{\prime \prime \prime}{ }_{s} \rho_{i}\right] \exp \left[\frac{-i q_{s}^{\prime \prime \prime 2} z_{1}}{z k_{s}}\right],
$$

where the terms depending on the variables $\rho_{s}$ were omitted. Using the expressions for the field operators in Eq.(3), we have

$$
\begin{array}{r}
\left\langle v a c\left|\hat{E}^{(+)}\left(\rho_{s}\right) \hat{E}^{(+)}\left(\rho_{i}\right)\right| \psi\right\rangle= \\
\mathcal{A}_{2}\left(\rho^{\prime}{ }_{s}\right) \int d \rho^{\prime \prime}{ }_{i} \mathcal{A}_{1}\left(\rho^{\prime \prime \prime}{ }_{i}\right) \int d q^{\prime \prime \prime}{ }_{s} \int d q^{\prime \prime \prime \prime \prime}{ }_{i} \exp \left[\frac{-i q_{s}^{\prime \prime \prime 2} z_{1}}{z k_{s}}\right] \exp \left[-i q^{\prime \prime \prime}{ }_{s} \cdot \rho^{\prime}{ }_{s}\right] \\
\times \exp \left[-i \rho^{\prime \prime \prime}{ }_{i}\left(\frac{\rho_{i} k_{i}}{f}+q^{\prime \prime \prime \prime}{ }_{i}\right)\right] \mathcal{V}\left(q^{\prime \prime \prime}{ }_{s}+q^{\prime \prime \prime \prime \prime}{ }_{i}\right),
\end{array}
$$

where $\mathcal{V}\left(q^{\prime \prime \prime}{ }_{s}+q^{\prime \prime \prime \prime}{ }_{i}\right)$ is the angular spectrum of the pump beam, given in Eq.(21). Again it is assumed that the signal and idler beams have the same wavelength. Using the change of 
variables defined in Eq.(13) we can rewrite Eq.(21) as

$$
\begin{array}{r}
\left\langle v a c\left|\hat{E}^{(+)}\left(\rho_{s}\right) \hat{E}^{(+)}\left(\rho_{i}\right)\right| \psi\right\rangle= \\
\mathcal{A}_{2}\left(\rho^{\prime}{ }_{s}\right) \int d \rho^{\prime \prime}{ }_{i} \mathcal{A}_{1}\left(\rho^{\prime \prime}{ }_{i}\right) \int d u \int d v \exp \left[-i \rho^{\prime \prime}{ }_{i} \frac{u+v}{2}\right] \\
\times \exp \left[i \frac{f}{2 k}(u v)\right] \exp \left[i \rho^{\prime}{ }_{s}\left(\frac{u-v}{2}\right)\right] \\
\times \exp \left[i \rho_{i}^{\prime \prime 2} \frac{k}{2 f}\right] \exp \left[-i \rho^{\prime \prime} \frac{\rho_{i} k_{i}}{f}\right] \mathcal{V}(u) .
\end{array}
$$

Solving integrals over $u$ and $v$, we have

$$
\begin{array}{r}
\left\langle\operatorname{vac}\left|\hat{E}^{(+)}\left(\rho_{s}\right) \hat{E}^{(+)}\left(\rho_{i}\right)\right| \psi\right\rangle= \\
\mathcal{A}_{2}\left(-\rho^{\prime}{ }_{s}\right) \times \int d \rho^{\prime \prime}{ }_{i} \mathcal{A}_{1}\left(\rho^{\prime \prime}{ }_{i}\right) \mathcal{V}\left[\frac{k}{f}\left(\rho^{\prime \prime}{ }_{i}-\rho_{s}\right)\right] \exp \left[-i \frac{k \rho^{\prime \prime}{ }_{i} \rho_{i}}{f}\right] .
\end{array}
$$

Assuming that the pump beam is a plane wave, we can perform the replacement

$$
\mathcal{V}\left[\frac{k}{f}\left(\rho^{\prime \prime}{ }_{i}-\rho_{s}\right)\right] \rightarrow \delta\left(\rho^{\prime \prime}{ }_{i}-\rho_{s}\right),
$$

and we will have the following expression for the coincidence count rate

$$
C\left(\rho_{s}, \rho_{i}\right) \propto\left|\mathcal{A}_{2}\left(-\rho_{s}\right) \mathcal{A}_{1}\left(\rho_{s}\right)\right|^{2} .
$$

Again, $C\left(\rho_{s}, \rho_{i}\right)$ is given by the product of the transmission functions of the gratings.

\section{DISCUSSION AND CONCLUSIONS}

Let us discuss some aspects of the two configurations investigated. In the scheme using the transfer of the angular spectrum, called pump-idler setup, the basic idea is to prepare a state for the twin beams in which a spatial structure of a grating is written in the conditional spatial correlations. The moiré is completed by using another grating just before detection. In this case, there is a clear difference from the "usual" (using classical optics) moiré. Because of the frequency conversion, the conditional structure is about two times larger than that placed in the pump beam. This may represent an improvement over "usual" moiré, however the discussion about the improvements of a quantum setup over the classical moiré, is complicated by the large number of possible configurations for a moiré. A complete discussion is beyond the scope of this work. 
From Eq.(16), it is seen the conditional character of the coincidence spatial distribution, through the dependence on the sum of the coordinates of the signal and idler detectors.

The scheme using one grating in each one of the beams, called signal-idler, is easily understood in terms of the Klyshko's advanced wave picture[11]. Idler detector works as it was the light source, shining light onto the idler grating. The image of the grating is projected onto the signal grating, as if the light emitted" from the idler detector was reflected into the signal path by the non-linear crystal. Finally, a lens is used to image the plane of the signal grating onto the signal detection plane, resulting in the moiré. According to Eq.(25), the coincidence count rate depends only on the signal detector coordinates. This may seem surprising at first sight, but it can be understood from the advanced wave interpretation, because the idler detector, which is playing the role of the source, is placed at the focal plane of a lens. Therefore, this detector is not sensitive to position displacements of the idler detector. In Eq.(25), the detector has been considered a point detector, and because of the spatial Fourier transform realized by the lens, it is working as a filter for the spatial frequencies. In this scheme, possible improvements over classical moiré is less evident, however this does not mean that improvements are impossible.

In both pump-idler and signal-idler schemes, there is one important difference from any classical implementation. Because of the non-local correlations between signal and idler photons, the information concerning the moiré depends on both signal and idler detections. Therefore, one can delay the detection of one of the photons. In the meantime, the non detected photons will be in a superposition state, while in a classical scheme, this would not be possible.

In conclusion, we have presented the calculation of the coincidence count rate for two implementations of moiré fringes using quantum images. The theoretical results also bring to attention special features of each scheme, such as the conditional character of the scheme pump-idler and the dependence only on the idler coordinates for the signal-idler scheme. We believe our study will assist for practical use of the quantum moiré fringes.

\section{Acknowledgments}

We acknowledge Dr. S. P. Walborn for reading this manuscript, and the financial support provided by Brazilian agencies CNPq, PRONEX, CAPES, FAPERJ, FUJB and the 
Milenium Institute for Quantum Information.

[*] Corresponding author. E-mail address: phsr@if.ufrj.br

[1] M. I. Kolobov and I. V. Sokolov, Phys. Lett. A 140, 101(1989); M. I. Kolobov and I. V. Sokolov; Sov. Phys. JETP 69, 1097(1989); L. A. Lugiato and A. Gatti, Phys. Rev. Lett. 70, 3868(1993); P. H. Souto Ribeiro, S. Pádua, J. C. Machado da Silva and G. A. Barbosa, Phys. Rev. A 49, 4176 (1994). A. Gatti and L. A. Lugiato, Phys. Rev. A 52, 1675(1995); D. V. Strekalov, A. V. Sergienko, D. N. Klyshko, and Y. H. Shih, Phys. Rev. Lett. 74, 3600 (1995); T. B. Pittman, Y. H. Shih, D. V. Strekalov, and A. V. Sergienko, Phys. Rev. A 52, R3429 (1995); G. A. Barbosa; Phys. Rev. A 54, 4473(1996); W. A. T. Nogueira, S. P. Walborn, S. Pdua, and C. H. Monken Phys. Rev. Lett. 86, 4009-4012 (2001); J. C. Howell, R. S. Bennink, S. J. Bentley, and R. W. Boyd Phys. Rev. Lett. 92, 210403 (2004)

[2] I. F. Santos, M. A. Sagioro, C. H. Monken, and S. Pádua Phys. Rev. A 67, 033812 (2003).

[3] E. J. S. Fonseca, C. H. Monken, and S. Pdua Phys. Rev. Lett. 82, 2868-2871 (1999).

[4] E. M. Nagasako, S. J. Bentley, R. W. Boyd, and G. S. Agarwal Phys. Rev. A 64, 043802 (2001).

[5] M. D’Angelo, M. V. Chekhova, and Y. Shih Phys. Rev. Lett. 87, 013602 (2001).

[6] A. N. Boto, P. Kok, D. S. Abrams, S. L. Braunstein, C. P. Williams, and J. P. Dowling Phys. Rev. Lett. 85, 2733-2736 (2000); P. Kok, A. N. Boto, D. S. Abrams, C. P. Williams, S. L. Braunstein, and J. P. Dowling Phys. Rev. A 63, 063407 (2001).

[7] J.A.O. Huguenin, M.P. Almeida, P.H. Souto Ribeiro, A.Z. Khoury. Moiré patterns in quantum images. Preprint quant-ph/0412127 at http://mentor.lanl.gov (2004).

[8] C. H. Monken, P. H. S. Ribeiro, and S. Pdua Phys. Rev. A 57, 3123-3126 (1998).

[9] M. P. Almeida, P.H. Souto Ribeiro, Transmission of Quantum Images Through Long Distances . Preprint quant-ph/0312134 at http://mentor.lanl.gov (2003).

[10] See for example, E. J. S. Fonseca, P. H. Souto Ribeiro, S. Pdua, and C. H. Monken Phys. Rev. A 60, 1530-1533 (1999).

[11] D. N. Klyshko, Physics Letters A, Volume 132, Issues 6-7, Pages 299-304 (1988). 